\title{
Three-Dimensional Imaging of Phase Ordering in an Fe-Al Alloy by Bragg Ptychography
}

\author{
Chan Kim, ${ }^{1, *}$ Virginie Chamard ${ }^{2, \dagger}$ Jörg Hallmann, ${ }^{1}$ Thomas Roth, ${ }^{1, \$}$ Wei Lu, ${ }^{1}$ Ulrike Boesenberg, ${ }^{1}$ \\ Alexey Zozulya, ${ }^{1}$ Steven Leake, ${ }^{3}$ and Anders Madsen ${ }^{1, \S}$ \\ ${ }^{1}$ European X-Ray Free-Electron Laser Facility, Holzkoppel 4, 22869 Schenefeld, Germany \\ ${ }^{2}$ Aix-Marseille Univ, CNRS, Centrale Marseille, Institut Fresnel, Marseille, France \\ ${ }^{3}$ ESRF-The European Synchrotron, 71 Avenue des Martyrs, 38000 Grenoble, France
}

(Received 1 August 2018; published 19 December 2018)

\begin{abstract}
We show three-dimensional images of phase ordering in a $\mathrm{Fe}_{55} \mathrm{Al}_{45}$ alloy obtained by coherent $\mathrm{x}$-ray diffraction Bragg ptychography. Fe-Al alloys display ordered phases where the atoms organize on sublattices resulting in the emergence of otherwise forbidden superlattice reflections. The degeneracy of the ordering results in antiphase domain boundaries that, in addition to the general lattice strain, provide phase shifts of the diffracted beam depending on the reflection. The reconstructed phase images can be separated into components originating from $B 2$ phase domains and lattice strain by performing Bragg ptychography on both the (002) fundamental and the (001) superlattice reflections.
\end{abstract}

DOI: 10.1103/PhysRevLett.121.256101

Intermetallic alloys have long been of interest due to their physicochemical properties and applications, for instance concerning the catalytic activity [1-3]. Fe-Al alloys, in particular, have many applications, e.g., due to excellent oxidation resistance and rich magnetic properties [4-7]. Binary alloys can exhibit very rich phase diagrams depending on composition and temperature and several chemically ordered phases exist where one or both atomic components order on sublattices as opposed to disordered phases where the occupancy of lattice sites is random with a probability only determined by the stoichiometric composition. This order-disorder phase transition has attracted much fundamental interest since it is regarded as a model system for second order phase transitions and critical behavior, e.g., the Heisenberg antiferromagnet and the Ising model, see Ref. [8] and references therein. Highresolution $\mathrm{x}$-ray scattering has been instrumental in characterizing metallic alloys and determining their phase diagrams. In particular, the order-disorder phase transition is accessible by $\mathrm{x}$-ray diffraction which can measure both short- and long-range order behavior with high sensitivity. Also, dynamical scaling theory has been tested to determine the dynamic critical exponents [9-11]. However, a more detailed picture of the ordered phase, i.e., the antiphase domain (APD) structure originating from the degeneracy in choice of sublattice, have been difficult to obtain with $\mathrm{x}$-ray imaging. Dark-field TEM has been widely used to this end [12], but an intrinsic limitation due to the small mean free path of electrons prevents threedimensional imaging.

Coherent $\mathrm{x}$-ray diffraction imaging (CXDI) in Bragg geometry [13-19] offers both high spatial resolution combined with high strain sensitivity in three dimensions which, however, did not succeed so far in imaging crystalline materials with a strong phase shift of the diffracted beam as is the case for antiphase domain boundaries (ADBs). Stadler et al. [20] attempted Bragg CXDI on Fe-Al crystals but only saw hints of the underlying APD structure, restricted to two-dimensional projections. In this Letter, we present the visualization of APD structures in three dimensions together with the associated general lattice strain. This is achieved by Bragg ptychography [21-25], which has shown robustness in retrieving images of materials exhibiting strong phase shifts [26], allowing us to compare and combine 3D images obtained at two different reflections, a fundamental and a superlattice peak, but originating from the same crystal grain. The superlattice peak is sensitive to both antiphase domain boundaries and phase variations from lattice strain and tilts while the fundamental peak is only sensitive to strain and tilts. This provides a unique normalization by which those effects can be separated and ADBs are clearly visualized.

The three-dimensional $\mathrm{x}$-ray Bragg ptychography experiment was performed at beam line ID01 at ESRFthe European Synchrotron in Grenoble, France. A partially coherent $7 \mathrm{keV}(\lambda=1.77 \AA) \mathrm{x}$-ray beam was focused by a Fresnel zone plate (FZP) with $300 \mu \mathrm{m}$ diameter and $70 \mathrm{~nm}$ outermost zone width. The $\mathrm{x}$-ray beam illuminated $60 \mu \mathrm{m}(\mathrm{H}) \times 300 \mu \mathrm{m}(\mathrm{V})$ (slit defined) of the FZP and was focused to about $180 \mathrm{~nm}(\mathrm{H}) \times 70 \mathrm{~nm}(\mathrm{~V})$ on the sample with a focal length of $118.6 \mathrm{~mm}$. To determine the illumination function of the sample, the x-ray beam was measured by an Andor CCD detector with $2560(\mathrm{H}) \times$ $2160(\mathrm{~V})$ pixels and $6.5 \mu \mathrm{m}$ pixel size located about $5 \mathrm{~m}$ downstream from the focal point, following the procedure described in Refs. [27,28] and detailed in the Supplemental Material, Sec. I [29]. The illumination function was characterized several times during the experiment to ensure 


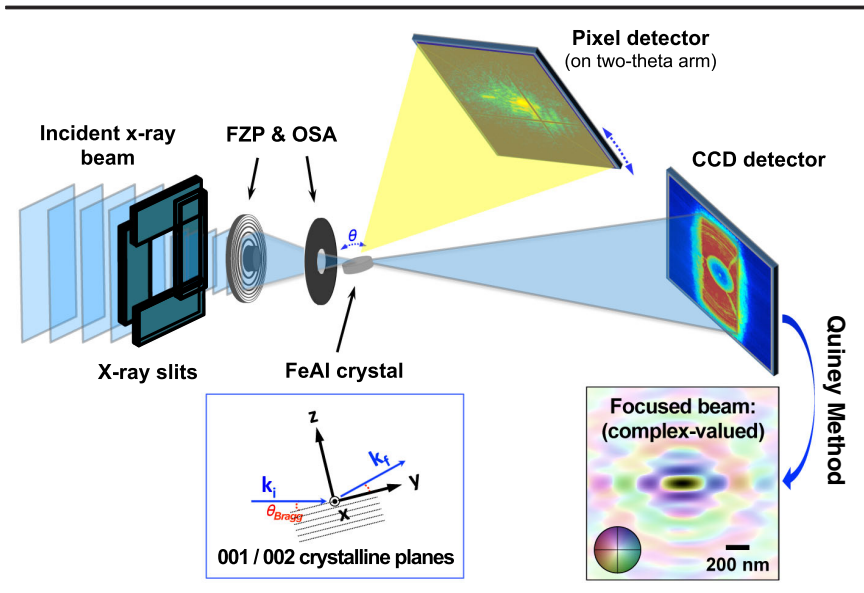

FIG. 1. Sketch of the Bragg ptychography experiment. A partially coherent x-ray beam is defined by slits and focused onto the sample by a Fresnel zone plate (FZP) with an order sorting aperture (OSA). The intensity scattered by the sample in Bragg condition is measured by a pixel detector over a range of $\theta$ rotation of the sample (rocking scan). This translates the detector plane through the crystal grain recording a $2 \mathrm{D}$ slice of the $3 \mathrm{D}$ intensity distribution for every $\theta$ angle. In addition, for every angular position overlapping exposures (ptychography) are taken by moving the sample ( $x-y$ surface coordinates) relative to the beam. If the sample is removed, the defocused $x$-ray beam can be measured by a CCD detector placed far downstream and the intensity in the focal point retrieved (Quiney method, bottom right).

stable focus conditions. This is of prime importance given the long acquisition times of the data series, about $10 \mathrm{~h}$ per each reflection. The Fe-Al crystal was mounted on a goniometer, translated into the focal spot and oriented with respect to the Bragg reflections. A suitable crystal grain was selected, so that all its diffraction components could be measured within the limited detector solid angle for a reasonable angular pitch along the rocking curve. Ptychographic 3D data sets were measured by a MAXIPIX pixel detector with $512 \times 512$ pixels of $55 \mu \mathrm{m}$ pixel size. The detector was mounted on the two-theta diffractometer arm in vertical scattering geometry with a distance of $1.2 \mathrm{~m}$ to the sample. The experimental setup is sketched in Fig. 1.

The $\mathrm{Fe}_{55} \mathrm{Al}_{45}$ sample was grown by MaTecK $\mathrm{GmbH}$ as a disk shaped bulk crystal with about $6 \mathrm{~mm}$ diameter and $2 \mathrm{~mm}$ thickness. Prior to the experiment, the crystal was annealed at $400{ }^{\circ} \mathrm{C}$ for several days under vacuum to stabilize the ordered $B 2$ phase characteristic of Fe rich $\mathrm{Fe}-\mathrm{Al}$ alloys. The crystal lattice is bcc with a mosaicity of $\sim 2^{\circ}$ and the surface normal corresponds to the average $\langle 001\rangle$ direction within an accuracy of $\sim 1^{\circ}$. The ordered phase is illustrated in Fig. 2. A bcc lattice can be thought of as two interpenetrating simple cubic sublattices and in the $B 2$ phase $\mathrm{Fe}$ occupies one of the sublattices. The other sublattice mostly consists of $\mathrm{Al}$ atoms but with some Fe present due to the slight off-stoichiometric conditions. This triggers the formation of ordered domains where
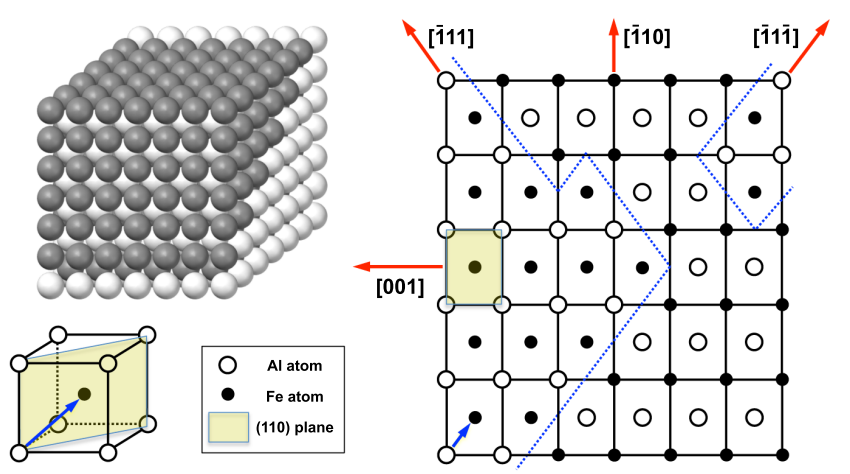

FIG. 2. Top left: schematic of bcc structure with antiphase domain boundaries (ADBs). Bottom left: general bcc structure. The blue arrow shows $\left\langle\frac{1}{2} \frac{1}{2} \frac{1}{2}\right\rangle$ which is the nearest neighbor direction where same-element atoms may become neighbors and create an ADB. Right: (110) plane with possible ADB intersections (dotted blue lines).

same-element atoms occasionally will be nearest neighbors due to the twofold degeneracy in choice of sublattice and hence ADBs will emerge as $R_{\mathrm{ADB}}=\left\langle\frac{1}{2} \frac{1}{2} \frac{1}{2}\right\rangle$ shifts in the (110) planes [20,30,31]. As a result of the ordering a (001) superlattice reflection emerges with a strength proportional to the absolute square of the difference between the atomic scattering factors of $\mathrm{Fe}$ and $\mathrm{Al}$. In the presence of an $\mathrm{ADB}$, the $\mathbf{Q}=(001)$ reflection experiences a phase shift $\phi=2 \pi\left(\mathbf{Q} \cdot \mathbf{R}_{\mathrm{ADB}}\right)=\pi$. The fundamental bcc reflection (002) exhibits a twice as large phase shift $(\phi=2 \pi)$ and is therefore insensitive to $B 2$ type ADBs. Both reflections will pick up phase shifts from lattice strain so to separate the effects, reconstructed (002) phase images will be used to normalize the (001) images thereby visualizing the ADB structure. Consequently, the success of the experiment relies on probing both a fundamental Bragg and a superlattice reflection of the exact same crystal grain.

The repeatability and possible drifts of the sample scanning stages were carefully monitored to ensure highquality data. A drift correction was implemented as described in the Supplemental Material, Sec. I [29]. Furthermore, to probe the exact same region of the crystal when switching between the (001) and the (002) Bragg peaks, 2D integrated intensity mesh scans [32] were performed, allowing a comparison between the scattered intensity for both reflections, identifying areas with similar features, see Fig. 3. This approach remains challenging for determining exactly the overlapping regions because contrast mechanisms, penetration depths, and projected volumes are different for the two reflections. Once a suitable region was identified, a switching procedure between (001) and (002) was set up to come back to the exact same position. The sphere of confusion of the diffractometer is much bigger than the beam size and the scanned region but-making use of the good repeatability of the instrument-a procedure could, nevertheless, be established which is further described in the Supplemental Material, Fig. 2 [29]. 


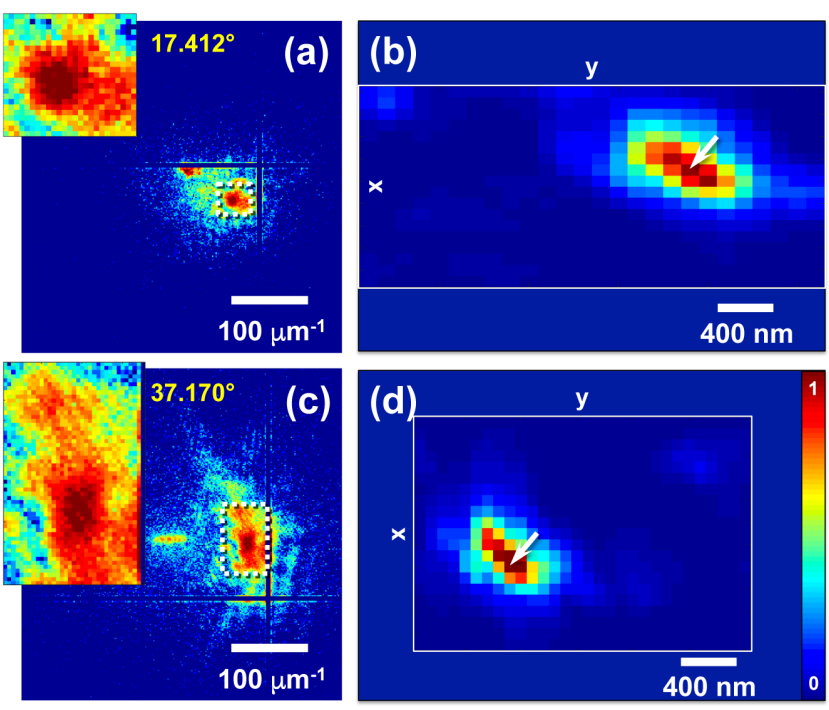

FIG. 3. 2D detector images (a),(c) and integrated intensity maps (b),(d) at $17.412^{\circ}(001)$ and $37.178^{\circ}$ (002) (vicinity of Bragg peaks). An area of reciprocal space, marked by white dotted rectangles in (a),(c), was selected for intensity integration to generate the maps shown in (b),(d). The white arrows in (b),(d) indicate the pixel corresponding to the detector images (a) and (c). Map (b) appears elongated in the $y$ direction compared with map (d) due to the difference in incidence angles. The "islands" of scattering in (b) and (d) originates from the same crystallite and this marker allows us to overlay data from (001) and (002) measurements.

3D Bragg ptychography data sets were measured at the (001) and (002) Bragg peaks with $\theta_{001}=17.547^{\circ}$ and $\theta_{002}=37.359^{\circ}$. For the $3 \mathrm{D}$ scanning, we used three motors: one angular $(\theta)$ and two translations $(x-y$ piezo scanner mounted on top of a hexapod table). The scan parameters were carefully selected to account for beam asymmetry, the projection geometry, and the smallest speckle size (see Supplemental Material, Sec. I [29]). For every $(x, y)$ position of the beam a high resolution $\theta$ scan was performed hence acquiring a pixel detector image of the scattering at every $(x, y, \theta)$ point. For both reflections this provides 3D data sets of approximately $40 \mathrm{~GB}$ to be analyzed with the inversion algorithms [25].

To accomplish the Bragg ptychography reconstructions a GPU-based algorithm was developed, introducing a 3D complex valued effective electron density to represent the diffracting sample structure [23-25]. The modulus is proportional to the electron density and the phase holds information about the lattice displacement field, e.g., strain, crystalline tilt, and potentially phase domain boundaries [for the (001) reflection only]. The lowest frequency of intensity variations in the $q_{z}$ direction of the (001) data is about $4.65 \mu \mathrm{m}^{-1}$ which corresponds to a thickness of about $1.35 \mu \mathrm{m}$. This is approximately what is expected as typical APD size of the sample after annealing [20] and it falls below the longitudinal coherence limit of this setup. This information was used for both data sets and introduced as a regularization term in the inversion process favoring a confinement of the solution within a finite thickness support [25]. The reconstructions employed a total of 4000 iterations using a combination of two different methods namely the ordered subset and conjugated gradient algorithms. The former has a faster convergence while the latter serves to stabilize the reconstruction [33]. A modulus homogenization $(\mathrm{MH})$ constraint was also employed in the second half of the reconstruction cycle. Indeed, the limited dynamical range of the intensity data sets gives rise to a bandwidth-limited reconstructed image, which presents strong modulus variations at regions where strong phase shifts occur, due to large lattice strain, crystal tilts, and in the (001) case also ADBs [34,35]. This interplay between the phase and the modulus can be alleviated by the introduction of a priori information such as the $\mathrm{MH}$ constraint [24]. By forcing the modulus to attain a more realistic value, the $\mathrm{MH}$ constraint helps in retrieving accurate phase values. The entire reconstruction was performed in the reciprocal space spanned by the detector frames and the conjugated real space.

After 4000 iterations the reconstruction was stabilized to about $\sim 0.5 \%$ variation. Phase ramp corrections [36,37] and coordinate transformations were performed as well as voxel size corrections (binning and interpolation) to convert images into the laboratory frame $(x, y, z)$ representation (see also Supplemental Material, Sec. II [29]). The final voxel size of the retrieved images is $10 \mathrm{~nm}(x) \times$ $26 \mathrm{~nm}(y) \times 10 \mathrm{~nm}(z)$ with a total of $522 \times 198 \times 150$ voxels for both the (001) and (002) data.

Because of the ADB contrast mechanism, the reconstructed (001) phase image $\left(\phi_{001}\right)$ is sensitive to both $\mathrm{ADBs}$ and phase variations originating from lattice displacement field of the crystal. The (002) phase image $\left(\phi_{002}\right)$ is only sensitive to the lattice displacement field and can hence be used to normalize the (001) image. More specifically, the phase shift from lattice displacement is proportional to the magnitude of the momentum transfer $|\mathbf{Q}|$ and here $\left|\mathbf{Q}_{(\mathbf{0 0 2})}\right|=2\left|\mathbf{Q}_{(\mathbf{0 0 1})}\right|$. Accordingly, the following calculation:

$$
\phi_{\text {sub }} \equiv\left(\phi_{001}-\phi_{002} / 2\right)
$$

should provide phase images presenting only the features characteristic of the phase ordering. This of course requires the subtraction to be done pixel by pixel, i.e., that the alignment of the two maps are accurate within $\sim 10 \mathrm{~nm}$ tolerance. Given the slow drift and alignment corrections applied during the long scan $(10 \mathrm{~h})$, the precise alignment was verified after the image reconstructions by using an additional processing of the retrieved images. To this end a group of voxels, free of any $\pi$ phase shifts, was selected from the (001) image. A correlation analysis between the group of voxels was applied searching for maximum resemblance of the phases by shifting the images with 

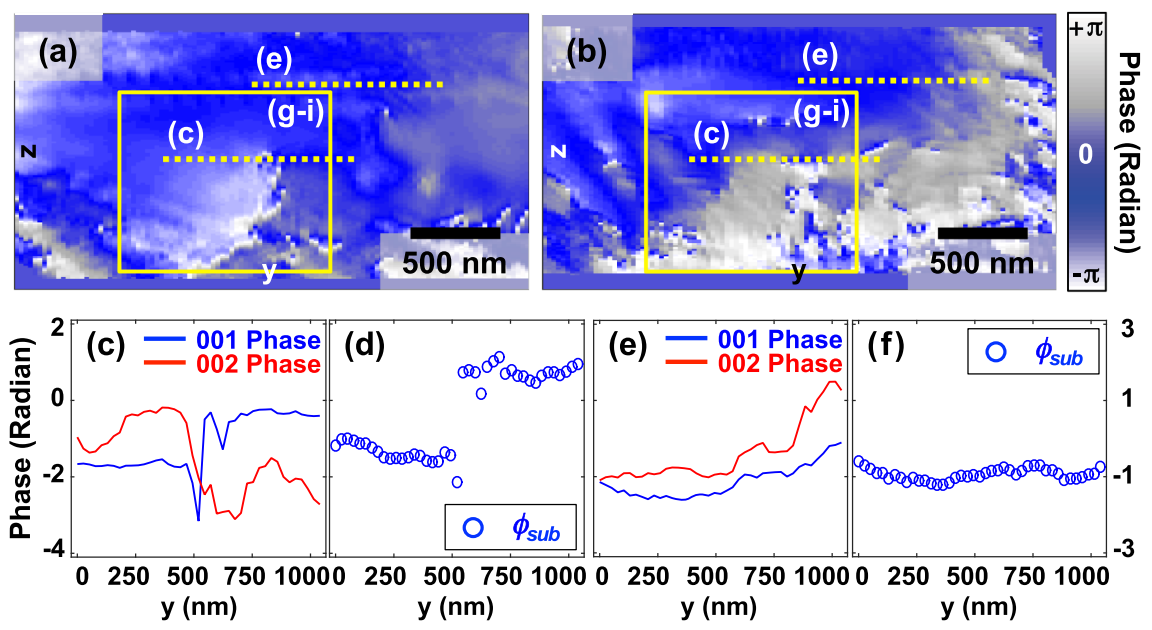
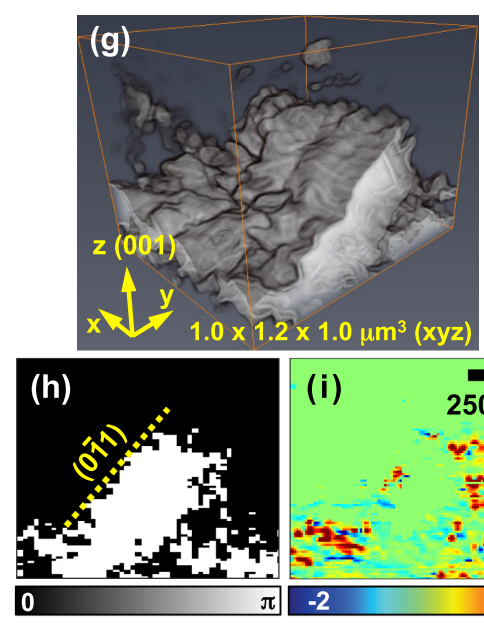

(i)

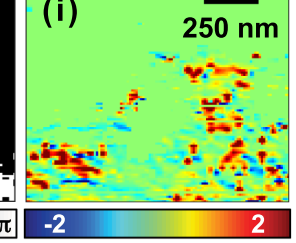

(002) Strain $\left(\times 10^{-3}\right)$

FIG. 4. (a),(b) Sectioned central slices of the reconstructed (001) phase (a) and (002) phase (b). (c),(e) Line profiles of (001) phase (blue lines) and (002) phase (red lines), which correspond to the yellow dotted lines in (a) and (b). (d),(f) Results of $\phi_{\text {sub }}$, calculated from the line profiles in (c) and (e). A strong $\pi$ phase shift from an ADB is visible in (d) while the profile in (f) has a constant phase. (g) 3D APD image in the selected region, yellow rectangles in (a) and (b). The empty area corresponds to one phase ordered domain and the gray colored area corresponds to another. The length of the coordinate system arrows is $250 \mathrm{~nm}$. (h), (i) $2 \mathrm{D}$ cuts showing a domain in the binary representation (h) and the corresponding (002) strain (i).

respect to each other in the $(x, y)$ plane. This procedure allows a final tweak of the adjustment between the two phase images and was necessary to avoid artifacts in image analysis. A more detailed explanation of this final optimization step can be found in the Supplemental Material, Sec. II [29]. No phase offset uncertainty was observed in the retrieved map which differs from the case of mosaic crystals measured with limited signal-to-noise ratio, where the phase relation in between the well-separated Bragg peaks is lost [25].

The subtraction procedure expressed in Eq. (1) can now be applied to normalize out the lattice displacement in the (001) image and highlight the phase ordering after a zero level correction was applied [38]. This allows us to emphasize the relative phase differences that matter here and in particular searching for $\pi$ phase shifts pointing towards the presence of ADBs. Two dimensional sections of the 3D (001) and (002) phase images showing the exact same image slice (central slice along the $x$ axis) are illustrated in Figs. 4(a) and 4(b). To highlight the phase structure the lattice displacement was subtracted using the procedure described above. Two line profiles from each reflection, marked as (c) and (e), are selected for illustration and the phase variations plotted in panels Figs. 4(c) and 4(e). $\phi_{\text {sub }}$ was then calculated from the line profiles as shown in Figs. 4(d) and 4(f). The (c) and (e) profiles have clear phase steps in both the (001) and (002) reconstructions. However, only case (c) points towards an ADB, with a clear $\pi$ phase jump in $\phi_{\text {sub }}$ (d). In (e) the phase jumps are clearly correlated and subtracts away in $\phi_{\text {sub }}$ (f) so this phase variation is solely related to lattice displacements. In order to ease the visualization of the 3D APD structure a binary representation was created with a certain threshold level denoted $\alpha$. The definition of the binary color map is

$$
\begin{array}{cc}
\pi-\alpha<\phi_{\text {sub }}<\pi+\alpha, & \phi_{\text {sub }}=\pi, \\
\text { otherwise, } & \phi_{\text {sub }}=0,
\end{array}
$$

when the phase values range from 0 to $2 \pi$. $\alpha=0.44 \pi$ was selected as a compromise between a sharp domain wall representation (small $\alpha$ ) and a visually clear image emphasizing the antiphase domain structure (large $\alpha$ ). The procedure to determine $\alpha$ is described in the Supplemental Material, Sec. III [29]. We note that the rather large value used for $\alpha$ arises from the noise further amplified in $\phi_{\text {sub }}$. Using the binary color map the APD structure close to an ADB can be illustrated, as shown in Fig. 4(g). Empty voxels correspond to one domain region while gray colored ones correspond to another. The $\mathrm{ADB}$ is the interface between these two volumes, corresponding to the occurrence of the $\pi$ phase shift.

In the $B 2$-ordered phase the domain walls emerge due to the possibility of having atoms of the same element as nearest neighbors, for instance in the (110) plane where $\left\langle\frac{1}{2} \frac{1}{2} \frac{1}{2}\right\rangle$ is the ADB displacement vector. Hence, the ADBs occur preferentially on $\{110\}$ planes which leads to $45^{\circ}$ tilted structures in a $(x, y, z)$ representation when $Q_{(001)}$ is the scattering vector (see Supplemental Material, Sec. III [29]). Figure 4(h) is a binary map showing a cross section of the 3D antiphase domain structure in panel (g). It emphasizes the shape of the antiphase domain. Furthermore, the comparison of this map with the strain 
map distribution extracted from the lattice displacement arising from the (002) fundamental reflection offers a unique possibility to study the correlation between lattice strain and ADB. To this aim, the exact same cross section of the reconstructed phase from the (002) reflection is shown in Fig. 4(i) converted to lattice strain, see Supplemental Material, Fig. 8 [29]. A strong correlation between the general lattice strain and the position of the domain wall is visible, particularly when the ADB is in a direction that requires rearrangements of atoms beyond the nearest neighbor $\left\langle\frac{1}{2} \frac{1}{2} \frac{1}{2}\right\rangle$ direction. In the preferred domain wall direction, indicated by the dashed line in Fig. 4(h), there is clearly less strain visible in Fig. 4(i). Hence, our results suggest that the closed loop ADB structures that have been observed previously [30], and which are imaged here for the first time in 3D, are mediated by crystal lattice strain. Other studies have reported (Ref. [20], and references therein) that in crystals of small strain the ADBs are often line shaped and form predominantly near $\{110\}$ planes.

In summary, we have successfully visualized the threedimensional $B 2$ antiphase-domain structure in an annealed iron-rich $\mathrm{Fe}-\mathrm{Al}$ alloy crystal. Bragg ptychography performed on two different reflections, (001) and (002) with different contrast mechanisms but originating from the same crystal grain, allows separation of phase domains and lattice displacement for the first time. We see that the ADBs predominantly align with $\{110\}$ planes. Sometimes closed structures are formed yielding nicely separated antiphase domains as the one we have imaged here in detail. In this case, there appears to be a strong correlation between general lattice strain and the location of the domain wall, particularly when it is not parallel to a $\{110\}$ plane. We speculate that the lattice defects induce the formation of these 3D structures. From 2D methods it is known that ADBs in Fe-Al crystals with small strain mostly appear as lines that begin and terminate at point defects [20]. Unfortunately, the resolution of this ptychography experiment is not sufficient to determine the exact size of an ADB. Often, we cannot resolve the phase jumps with the $10 \mathrm{~nm}$ voxel size achieved here. To improve the resolution and phase sensitivity more coherent flux or longer data acquisitions would be required. However, the possibility of performing longer acquisitions is challenged by a limitation arising from the nanometer stability required in Bragg ptychography on multiple reflections. Some improvements regarding the inversion algorithms are currently investigated, including sparse angular step, angular uncertainties, and probe retrieval. We expect that the next generation of diffraction limited synchrotron sources, delivering 10-100 times more coherent flux than current forefront storage rings, will improve the conditions for these types of experiment and atomic resolution can be approached. Alternatively, coherent diffraction imaging of APD structures using x-ray free-electron lasers could benefit from the huge coherent flux if the sample damage issue can be mitigated.
We acknowledge ESRF-the European Synchrotron for provision of beam time at the ID01 beam line. Tobias Schulli, Carsten Richter, and Vincent Favre-Nicolin (ESRF), Marc Allain (CNRS, Institut Fresnel), Juliane Reinhardt, Andreas Stierle, and Vedran Vonk (DESY), Klaus Giewekemeyer, Roman Shayduk, Johannes Möller, Markus Scholz, and Mario Reiser (European XFEL) are acknowledged for helpful discussions and support.

*chan.kim@xfel.eu

virginie.chamard@fresnel.fr

*Present address: ESRF-The European Synchrotron, 71 Avenue des Martyrs, 38000 Grenoble, France.

§anders.madsen@xfel.eu

[1] F. Besenbacher et al., Science 279, 1913 (1998).

[2] V. R. Stamenkovic, B. Fowler, B. S. Mun, G. Wang, P. N. Ross, C. A. Lucas, and N. M. Markovic, Science 315, 493 (2007).

[3] H. C. Kang et al., Nanoscale 5, 7184 (2013).

[4] F. Plazaola, E. Apiñaniz, D. M. Rodriguez, E. Legarra, and J.S. Garitaonandia, in Advanced Magnetic Materials, edited by L. Malkinski (InTech, London, 2012), p. 133.

[5] D. M. Rodriguez, E. Apiñaniz, F. Plazaola, J. S. Garitaonandia, J. A. Jiménez, D. S. Schmool, and G. J. Cuello, Phys. Rev. B 71, 212408 (2005).

[6] A. V. Smirnov, W. A. Shelton, and D. D. Johnson, Phys. Rev. B 71, 064408 (2005).

[7] E. Menéndez, J. Sort, M. O. Liedke, J. Fassbender, S. Suriñach, M. D. Baró, and J. Nogués, New J. Phys. 10, 103030 (2008).

[8] A. Madsen, J. Als-Nielsen, J. Hallmann, T. Roth, and W. Lu, Phys. Rev. B 94, 014111 (2016).

[9] S. Brauer, G. B. Stephenson, M. Sutton, R. Brüning, E. Dufresne, S. G. J. Mochrie, G. Grübel, J. Als-Nielsen, and D. L. Abernathy, Phys. Rev. Lett. 74, 2010 (1995).

[10] A. Fluerasu, M. Sutton, and E. M. Dufresne, Phys. Rev. Lett. 94, 055501 (2005).

[11] F. Livet, M. Fèvre, G. Beutier, and M. Sutton, Phys. Rev. B 92, 094102 (2015).

[12] W. Liu, A. Gemperle, J. Gemperlova, V. Paidar, and E. Nembach, Acta Mater. 46, 6173 (1998).

[13] I. Robinson and R. Harder, Nat. Mater. 8, 291 (2009).

[14] A. Yau, W. Cha, M. W. Kanan, G. B. Stephenson, and A. Ulvestad, Science 356, 739 (2017).

[15] K. M. Pavlov, V. I. Punegov, K. S. Morgan, G. Schmalz, and D. M. Paganin, Sci. Rep. 7, 1132 (2017).

[16] M. C. Newton, S. J. Leake, R. Harder, and I. K. Robinson, Nat. Mater. 9, 120 (2010).

[17] A. Ulvestad, J. N. Clark, R. Harder, I. K. Robinson, and O. G. Shpyrko, Nano Lett. 15, 4066 (2015).

[18] S. Labat et al., ACS Nano 9, 9210 (2015).

[19] F. Hofmann, N. W. Phillips, R. J. Harder, W. Liu, J. N. Clark, I. K. Robinson, and B. Abbey, J. Synchrotron Radiat. 24, 1048 (2017).

[20] L.-M. Stadler, R. Harder, I. K. Robinson, C. Rentenberger, H.-P. Karnthaler, B. Sepiol, and G. Vogl, Phys. Rev. B 76, 014204 (2007). 
[21] J. M. Rodenburg, A. C. Hurst, A. G. Cullis, B. R. Dobson, F. Pfeiffer, O. Bunk, C. David, K. Jefimovs, and I. Johnson, Phys. Rev. Lett. 98, 034801 (2007).

[22] P. Thibault, M. Dierolf, A. Menzel, O. Bunk, C. David, and F. Pfeiffer, Science 321, 379 (2008).

[23] P. Godard, G. Carbone, M. Allain, F. Mastropietro, G. Chen, L. Capello, A. Diaz, T.H. Metzger, J. Stangl, and V. Chamard, Nat. Commun. 2, 568 (2011).

[24] A. I. Pateras, M. Allain, P. Godard, L. Largeau, G. Patriarche, A. Talneau, K. Pantzas, M. Burghammer, A. A. Minkevich, and V. Chamard, Phys. Rev. B 92, 205305 (2015).

[25] F. Mastropietro, P. Godard, M. Burghammer, C. Chevallard, J. Daillant, J. Duboisset, M. Allain, P. Guenoun, J. Nouet, and V. Chamard, Nat. Mater. 16, 946 (2017).

[26] M. O. Hill et al., Nano Lett. 18, 811 (2018).

[27] H. M. Quiney, A. G. Peele, Z. Cai, D. Paterson, and K. A. Nugent, Nat. Phys. 2, 101 (2006).

[28] V. Chamard, M. Allain, P. Godard, A. Talneau, G. Patriarche, and M. Burghammer, Sci. Rep. 5, 9827 (2015).
[29] See Supplemental Material at http://link.aps.org/ supplemental/10.1103/PhysRevLett.121.256101 for further details about the experiment and data analysis.

[30] M. J. Marcinkowski and N. Brown, J. Appl. Phys. 33, 537 (1962).

[31] L.-M. Stadler, B. Sepiol, J. W. Kantelhardt, I. Zizak, G. Grübel, and G. Vogl, Phys. Rev. B 69, 224301 (2004).

[32] G. A. Chahine et al., J. Appl. Crystallogr. 47, 762 (2014).

[33] P. Godard, M. Allain, V. Chamard, and J. Rodenburg, Opt. Express 20, 25914 (2012).

[34] P. Godard, M. Allain, and V. Chamard, Phys. Rev. B 84, 144109 (2011).

[35] C. Kim, V. Chamard, J. Hallmann, W. Lu, U. Boesenberg, and A. Madsen, Microsc. Microanal. 24, 48 (2018).

[36] J. M. Bioucas-Dias and G. Valadão, IEEE Trans. Image Process. 16, 698 (2007).

[37] M. B. Luu et al., New J. Phys. 16, 093012 (2014).

[38] S. Marathe, S. S. Kim, S. N. Kim, C. Kim, H. C. Kang, P. V. Nickles, and D. Y. Noh, Opt. Express 18, 7253 (2010). 